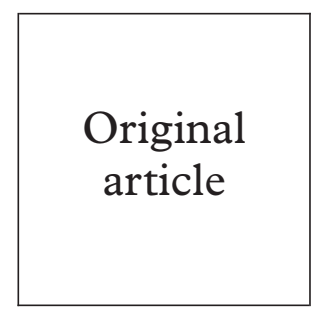

\section{An intervention study to reduce adverse pregnancy outcomes as a result of syphilis in Mozambique}

\author{
N Bique Osman, K Challis, E Folgosa, M Cotiro, S Bergström
}

\author{
Department of \\ Obstetrics and \\ Gynaecology, Eduardo \\ Mondlane University, \\ Central Hospital, \\ Maputo, Mozambique \\ $\mathrm{N}$ Bique Osman \\ M Cotiro \\ Division of \\ International Health \\ (IHCAR), Department \\ of Public Health \\ Sciences, Karolinska \\ Institutet, SE-171 76 \\ Stockholm, Sweden \\ $\mathrm{N}$ Bique Osman \\ $\mathrm{K}$ Challis \\ S Bergström \\ Department of \\ Obstetrics and \\ Gynaecology, \\ Sundsvall Hospital, \\ Sundsvall, Sweden \\ $\mathrm{K}$ Challis \\ Department of \\ Microbiology, Eduardo \\ Mondlane University, \\ Maputo, Mozambique \\ E Folgosa \\ Correspondence to: \\ Staffan Bergström \\ Staffan.Bergstrom@phs.ki.se \\ Accepted for publication \\ 25 February 2000
}

Objectives: To create and evaluate an alternative screening approach among pregnant women in order to reduce adverse pregnancy outcome as a result of syphilis in Mozambique.

Methods: Four suburban antenatal clinics, two "control" and two "intervention" clinics, were compared regarding syphilis screening and treatment. Pregnant women with positive rapid plasma reagin $(R P R)$ test $(n=929)$ were enrolled, 453 in the intervention and 476 in the control clinics. In control clinics the normal routine regarding syphilis screening was followed for 383 women remaining for follow up. In intervention clinics nurse midwives were trained to perform the RPR test. RPR seropositive cases were immediately treated on site by the nurse midwives and the partners were invited to come any afternoon for treatment. In the third trimester (around 30 weeks) a new RPR test was performed and all women with positive RPR test results were again treated and the partners were invited to come for treatment.

Results: At delivery, the drop out rate was $15.7 \%$ in the intervention and $20.1 \%$ in the control group. The perinatal mortality was significantly higher in the control group than in the intervention group, $3.4 \% v 1.3 \%(\mathrm{p}=0.030)$. At delivery the intervention group had significantly more negative RPR results- $40.9 \%$ v $24.2 \%(\mathrm{p}=0.000)$.

Conclusion: More active training of nurse midwives in antenatal care to perform on site RPR tests, to give syphilis treatment, and to notify partners results in improved perinatal outcome and more seronegative parturient women.

(Sex Transm Inf 2000;76:203-207)

Keywords: syphilis; intervention; pregnancy; Mozambique

\section{Introduction}

Syphilis is an important cause of morbidity and mortality during pregnancy in sub-Saharan African countries, where high rates of seropositivity have been found-for example, $4 \%$ in Kenya and Malawi, ${ }^{2}{ }^{2} 6-15 \%$ in South Africa, ${ }^{3-5} 8 \%$ in Zambia, ${ }^{6} 14 \%$ in Zimbabwe, ${ }^{7}$ and $5-15 \%$ in Mozambique. ${ }^{8-10}$

Previous studies on stillbirth in Mozambique, ${ }^{11-14}$ in Malawi, ${ }^{2}$ in South Africa, ${ }^{3}$ and in Zimbabwe $^{7}$ have shown that pregnant women with active syphilis during pregnancy are more likely to have a perinatal death. Another study in Maputo found an association between syphilis and mid-trimester miscarriage. $^{15}$ The syphilis seroprevalence among pregnant women has remained around 10$15 \%$ for the past 15 years. ${ }^{814}$ Although around $90 \%$ of pregnant women attend antenatal services and the ministry of health policy is to screen all pregnant women for syphilis, several barriers to its effective implementation have been recognised.

In the Nairobi decentralisation project, ${ }^{16}$ in which more than 13000 pregnant women were screened for syphilis with RPR (rapid plasma reagin) test done on site by $\mathrm{MCH}$ (maternal and child health) nurses, $87.3 \%$ of seroreactive women were treated immediately and $50 \%$ of partners were also treated. It might, theoretically, have prevented 413 cases of congenital syphilis at a cost of approximately $\$ 50$ per prevented case.
In the Zambian project ${ }^{6}$ the adverse outcomes attributable to syphilis were reduced to $28.3 \%$ in the intervention clinics as against $72.4 \%$ in the control clinics. The two Kenyan and Zambian studies have demonstrated that it is feasible and cost effective to invest in programmes in countries with high rates of syphilis. The purpose of this interventional antenatal care study was to create and evaluate an alternative screening approach among pregnant women in order to reduce adverse pregnancy outcomes as a result of syphilis in Mozambique.

\section{Subjects and methods}

Maputo is the national capital of Mozambique and has approximately 1.5 million inhabitants. Around $90 \%$ of pregnant women are enrolled in antenatal clinics. Two suburban antenatal clinics, Malhangalene and Primeiro de Maio, were selected for an intervention and another two similar, suburban antenatal clinics, Bagamoio and José Macamo, were selected for control purposes. For staff reasons it was considered impossible to randomise patients to one of the two treatment options in one and the same clinics. We therefore carried out the study in separate clinics assuming that the populations covered by the four clinics are comparable.

\section{CONTROL CLINICS}

At these clinics the normal routine regarding syphilis screening was followed. The nurse midwives sent all pregnant women coming to 
the antenatal clinic to the laboratory to have an RPR test done. The women had to return 1-2 days later for the result and had then to go back with the result to the nurse midwife, who had to send all positive results to the STD clinic or to a doctor for appropriate treatment. The routine treatment was three weekly doses $(2.4$ million IU) of benzathine penicillin but the compliance was generally poor for the three doses, estimated as less than $50 \%$ completing the treatment. Previously the benzathine penicillin was free of charge but currently the patients have to pay for it. In practice, no partner notification takes place. In the control clinics the nurse midwives were advised to obtain the women's acceptance to enter the study, to make a list of names and addresses of all positive RPR cases, to label their antenatal cards, and to recommend delivery at the Maputo Central Hospital. Owing to irregular supply of RPR reagents at the health centres, such reagents were provided by the project to the laboratory in order to enable testing of all pregnant women in the control clinics. Otherwise no interventions were made in these clinics. However, when half of the patients in the control clinics had been followed until birth we noted a low compliance. This necessitated an intervention to increase the compliance and a small material incentive was given to each of these women in order to enhance willingness to deliver in the central hospital.

INTERVENTION CLINICS

All the nurse midwives in these clinics had a refresher course regarding the importance of syphilis screening, diagnosis and treatment, patient counselling, and partner notification. After this course they were trained over 2 weeks to perform the RPR test at the STD national reference laboratory of the department of microbiology at the faculty of medicine. The clinics were supplied with needles, Vacutainer tubes and reagents for the RPR test as well as benzathine penicillin. From August 1996 to July 1998 all new antenatal attendees were offered RPR testing done by the nurse midwife after explanation about the study. The following method was used. From each woman, blood samples were collected by venepuncture to a Vacutainer tube. Serum aliquots were qualitatively (undiluted sera) tested, by the midwives, using the RPR card test (Immutrep RPR, Omega Diagnostics Limited, Scotland) according to the manufacturer's instructions, at the antenatal clinic. All sera giving a reactive (positive) result and non-reactive sera at the antenatal clinic were sent to the microbiology laboratory of the faculty of medicine, serving as reference laboratory. At the laboratory the samples were again qualitatively (undiluted sera) tested with RPR card test and with Treponema pallidum haemagglutination assay (TPHA) (Syphilia TPHA 200, Sanofi Diagnostics Pasteur, Marnes-la Coquette, France). All sera being positive in the RPR test were further analysed by serial twofold dilutions of serum in physiological saline up to $1: 1024$. The highest dilution showing flocculation was reported. To determine the serum end point titres to the haemagglutination assay, all sera reactive in the qualitative assay were quantitatively tested in twofold dilutions (1:80 up to 1:5160) in absorbing diluent (a mixture of sonicated cell membranes from sheep erythrocytes, normal rabbit testicular extract, sonicated Reiter treponemes, and normal rabbit serum).${ }^{13}$ Results were reported as the highest dilution giving haemagglutination.

At the antenatal clinics, the RPR seropositive cases were immediately treated with a single intramuscular dose of 2.4 million IU benzathine penicillin and were counselled to return for a repeat RPR test at 30 weeks. This patient counselling was intentionally individual and carried out in privacy. The partners were invited to come any afternoon as soon as possible to the antenatal clinic for treatment.

At the first visit the antenatal card was filled in with a complementary questionnaire regarding socioeconomic and physical characteristics, and obstetric history. The cards were labelled to recommend delivery at the Maputo Central Hospital in order to facilitate tracing of pregnancy outcomes. The subsequent visits were according to norm, but in the third trimester (around 30 weeks) a new RPR test was performed and all women with positive RPR test results were again treated with a single dose of 2.4 million IU benzathine penicillin and the partners were again invited to come in the afternoon for treatment.

\section{AT DELIVERY}

All pregnant women enrolled in the study from the intervention and control clinics were advised to deliver at the Maputo Central Hospital; serum aliquots were collected for a new qualitative and quantitative RPR test. A new TPHA test was performed at the reference laboratory of the faculty of medicine. The questionnaire regarding socioeconomic conditions, physical characteristics, and obstetric history was filled in at this time for the women in the control clinics.

\section{AFTER DELIVERY}

The newborns were examined and all antenatal cards were collected. Several home visits were performed to find women who did not come for delivery at the central hospital. When home visits failed local authorities were contacted to find them but we succeeded in finding few of them. Many of the enrolled women obviously had given wrong addresses, possibly owing to their unwillingness to be associated with this stigmatising disease.

\section{STATISTICAL METHODS}

The statistical analyses were performed with EPI-INFO and SPSS software. For continuous variables, mean values and $t$ test for equality of means were used, while for categorical variables, comparison of proportions $\chi^{2}$ test and, when applicable, the Fisher's exact test were used. p Values less than 0.05 were considered statistically significant. 
Intervention group

First RPR
(enrolment)
Second RPR
(follow up, ANC)

Third RPR

(delivery)

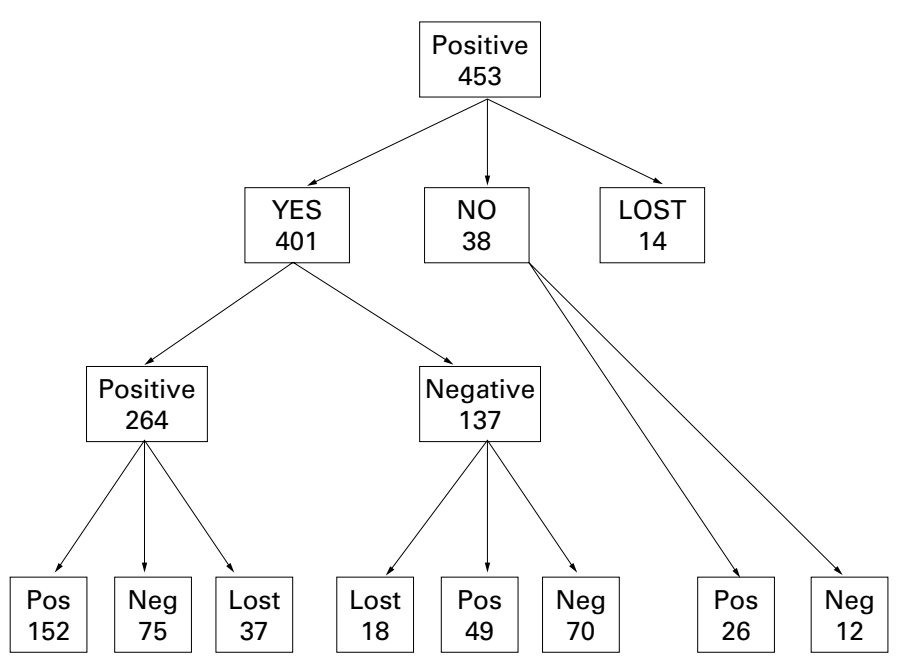

Total No of women

recovered at delivery $384<$ Positive 227

Negative 157

Total loss at delivery 69

Figure 1 Flow chart of 453 RPR positive pregnant women identified at the interventions clinics and followed for their second and third RPR test at the antenatal clinic and at delivery, respectively.

ETHICAL ISSUES

All women participating in the study gave oral consent to participate after the explanation about the objectives of the study. Written consent was not requested since women often refused to sign a document that indicated a sexually transmitted disease. The study was accepted by the ethics committee at the central hospital and medical faculty at Eduardo Mondlane University in Maputo.

\section{Results}

From August 1996 to July 1998, 453 pregnant women were enrolled in the intervention clinics, and from August 1996 to December 1998, 476 pregnant women in the control clinics, of whom 383 remained to be followed up (see figs 1 and 2). Very few women declined to participate. At delivery, 382 were found in intervention group and 305 in the control group resulting in a dropout rate of $15.7 \%$ in

\section{Control group}

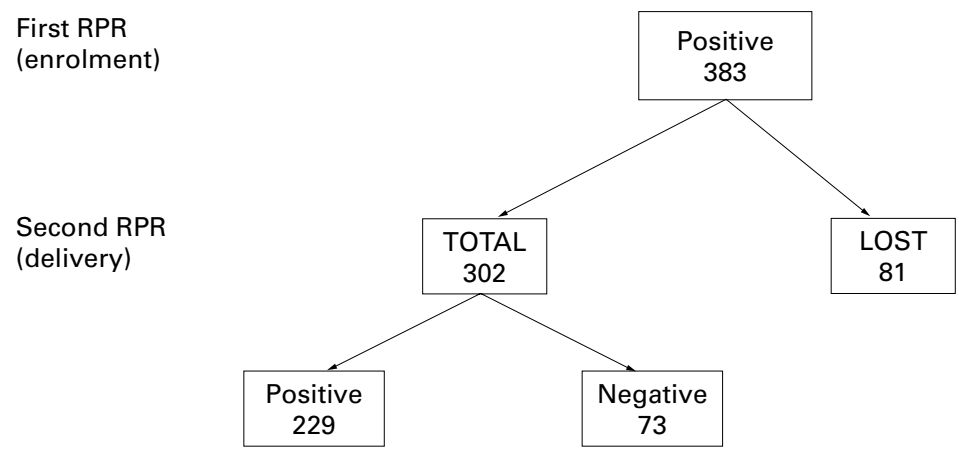

Figure 2 Flow chart of $383 R P R$ positive pregnant women identified at the control clinics and followed for their second RPR test at delivery. the intervention and $20.1 \%$ in the control group, respectively.

The mean age was similar in the two groups (23.6 years and 24.0 years, respectively), while some socioeconomic characteristics (schooling, household size, housing, and alleged professions) showed slight differences. The mean parity was 1.9 in the intervention group and 1.7 in the control group. No significant differences between intervention and control groups were noted regarding history of stillbirths and live births and number of currently living children.

Past history of STDs was reported in $15.7 \%$ of the intervention group, while it was $28.7 \%$ in the control group. Information was missing in $0.4 \%$ of the intervention group and in $16.7 \%$ of the control group. Only $1.1 \%$ in the intervention group and $2.1 \%$ in the control group admitted having more than one partner but the information was often missing in the control group $(48.6 \% \quad v 8.2 \%$ in the intervention group).

The mean gestational age at first visit was 20.7 weeks in intervention group and 20.3 weeks in control group $(\mathrm{p}=0.425)$. The mean number of visits was 4.5 in the intervention group and 4.9 in the control group. The mean

Table 1 Pregnancy outcome in the intervention and control groups

\begin{tabular}{|c|c|c|c|c|c|}
\hline \multirow{2}{*}{$\begin{array}{l}\text { Pregnancy } \\
\text { outcome }\end{array}$} & \multicolumn{2}{|c|}{ Intervention } & \multicolumn{2}{|c|}{ Control } & \multirow[b]{2}{*}{$p$ Value } \\
\hline & No & $\%$ & No & $\%$ & \\
\hline Abortion & 6 & 1.5 & 0 & 0 & 0.03 \\
\hline Preterm & 34 & 8.6 & 33 & 10.5 & 0.54 \\
\hline Term & 338 & 85.8 & 272 & 86.9 & 0.75 \\
\hline Post-term & 16 & 4.1 & 8 & 2.6 & 0.37 \\
\hline Subtotal & 394 & 100.0 & 313 & 100.0 & \\
\hline Missing & 59 & & 70 & & \\
\hline
\end{tabular}


Table 2 Neonatal outcome in the intervention and control groups

\begin{tabular}{|c|c|c|c|c|c|}
\hline \multirow[b]{2}{*}{ Neonatal outcome } & \multicolumn{2}{|c|}{ Intervention } & \multicolumn{2}{|c|}{ Control } & \multirow[b]{2}{*}{$p$ Value } \\
\hline & No & $\%$ & No & $\%$ & \\
\hline Alive and healthy & 365 & 95.5 & 275 & 90.2 & 0.008 \\
\hline Alive after care in neonatal ward & 12 & 3.1 & 22 & 7.2 & 0.023 \\
\hline Alive on the 7 th day & 377 & 98.7 & 292 & 95.8 & 0.030 \\
\hline Intrauterine death & 5 & 1.3 & 8 & 2.6 & 0.159 \\
\hline Subtotal & 382 & 100.0 & 305 & 100.0 & \\
\hline Missing & 71 & & 78 & & \\
\hline
\end{tabular}

gestational age at delivery was 38.7 and 38.2 weeks respectively, and alleged risk factors in the antenatal card were present in $22.1 \%$ in the intervention clinics and $5.8 \%$ in the control clinics but missing cases were $8.6 \%$ in the intervention clinics and $33.7 \%$ in the control clinics.

Different pregnancy outcomes such as abortion, gestational length at delivery are presented in table 1 . The perinatal mortality was significantly higher in the control group $(p=0.030)$ (table 2). The reported prevalence of abortion was higher in the intervention group (1.5 $v 0 \%)$. This difference should be interpreted with caution, since there were $70 / 383(18.3 \%)$ control women who did not report pregnancy outcome. It is possible that a substantial proportion of them had adverse pregnancy outcomes. Neonatal infection affected newborns in the control group about seven times more often than newborns in the intervention group $(\mathrm{p}=0.06)$.

In the intervention clinic, one aim was to address the issue of RPR quality performed by nurse midwives in comparison with the quality of the same test performed by laboratory technicians at the microbiology reference laboratory. The nurses sent 1069 negative sera, of which 62 were positive at the reference laboratory, giving $5.8 \%$ false negative results. Of 431 positive sera sent by the nurses, 55 were negative at the reference laboratory, giving $12.8 \%$ false positive results.

In the third trimester, a second RPR test was performed among women in the intervention group. Of all 453 women in this group, there were $52(11.5 \%)$ missing in the third trimester and $137 / 401(34.1 \%)$ had negative results and $264 / 401(65.9 \%)$ had positive results. Out of these 264 women with positive RPR in the third trimester, $75(28.4 \%)$ were negative at birth and $152(57.6 \%)$ remained positive at birth. Of 137 women being negative in the third trimester, $70(58.8 \%)$ remained negative at birth and $49(41.2 \%)$ became positive at

Table 3 RPR seroreversion rates at delivery (reactive to non-reactive test) in the intervention and control groups

\begin{tabular}{|c|c|c|c|c|c|}
\hline & \multicolumn{2}{|c|}{ Intervention } & \multicolumn{2}{|c|}{ Control } & \multirow[b]{2}{*}{ p Value } \\
\hline & No & $\%$ & No & $\%$ & \\
\hline \multicolumn{6}{|c|}{ RPR at delivery } \\
\hline Negative & 157 & 40.9 & 73 & 24.2 & 0.000 \\
\hline Positive & 227 & 59.1 & 229 & 75.8 & \\
\hline Subtotal & 384 & 100.0 & 302 & 100.0 & \\
\hline Missing & 69 & & 81 & & \\
\hline \multicolumn{6}{|c|}{ TPHA at delivery } \\
\hline Negative & 123 & 34.2 & 74 & 25.0 & 0.013 \\
\hline Positive & 237 & 65.8 & 222 & 75.0 & \\
\hline Subtotal & 360 & 100.0 & 297 & 100.0 & \\
\hline Missing & 93 & & 87 & & \\
\hline
\end{tabular}

birth. These 49 women may have been reinfected.

Partner treatment in the intervention clinics was successful in $76 \%$ of partners invited; these men received the first treatment and $40 \%$ received the second treatment. In the control clinics partner notification was virtually nonexistent. At delivery RPR and TPHA were performed in women of both groups and the intervention group had significantly more negative results (table 3). Titres above 1/16 were found in $8.1 \%$ of the control group and in $10.3 \%$ of the intervention group (difference not significant).

\section{Discussion}

The results of this study show that it is feasible to have a syphilis screening programme for all pregnant women attending the antenatal clinics in a low income setting, where the nurse midwives perform an RPR test, interpret the test, and give immediate treatment as well as counselling and partner notification. The fact that the study was not randomised would not seem to invalidate this results, since the populations covered by the four clinics involved were similar. With this intervention it was possible to reduce maternal seropositivity at delivery and the perinatal death rate but we could not show a decrease in other adverse outcomes such as abortion and low birth weight. One explanation might be the high dropout rate in the control group, implying a possible loss of women with adverse pregnancy outcomes who might not be motivated to come back. The stillbirth rate in the intervention group was only half of the rate in the control group but this difference is not statistically significant since the rates are low, but not having a child alive on the seventh day post partum is significantly more common in the control group. The advantages of such on site screening for maternal syphilis with RPR in a setting similar to the one studied here have also been described by other authors. ${ }^{16-18}$

RPR and VDRL are non-treponemal tests used for screening and it is recommended for the positive results to perform more specific tests such as the MHA-TP or FTA-ABS tests for antitreponemal antibodies. ${ }^{19}$ In developing countries there are many antenatal clinics without laboratory facilities and the laboratories have many constraints and shortage of reagents is usual. RPR is a simple test which can be performed without a microscope by any trained and motivated person.

Comparing the RPR results from the nurses midwives and from the reference laboratory, there were $5.8 \%$ false negatives and $12.1 \%$ false positives. These results are acceptable for nurses midwives if we compare them with other studies. In a previous study done in eight provinces in Mozambique by Liljestrand et al, VDRL tests done by district hospital laboratories were compared with tests carried out in a reference laboratory of the faculty of medicine in Maputo and $51 \%$ false positive and $44 \%$ false negative results were found. ${ }^{8}$ In the control clinics the quality of RPR test was not checked as that would have implied an undue 
intervention. These control clinics were, however, better than an average antenatal clinic, since we provided a regular supply of RPR reagents.

In affluent countries such as Canada, ${ }^{19}$ France, ${ }^{20}$ and the United Kingdom ${ }^{21}$ maternal and congenital syphilis has a low prevalence but screening is still recommended. A cost benefit analysis performed in the United Kingdom concluded that antenatal screening remained worthwhile. ${ }^{22}$ Similarly, in the United States there was an increase in syphilis cases beginning in the late $1980 \mathrm{~s}$, still being prominent in the heterosexual population ${ }^{23}$ and congenital syphilis is increasing in some American regions. ${ }^{24}$ US health authorities recommend screening at enrolment, in the third trimester, and at delivery.

In low income countries in sub-Saharan Africa several studies have shown major deficiency in syphilis screening and treatment. ${ }^{137925}$ In a previous cohort study (Bique Osman et al, unpublished) $58 \%$ of pregnant women did not have a syphilis test performed.

In this intervention we adopted the recommendation of Centers for Disease Control and Prevention (CDC) regarding screening and treatment of maternal syphilis. For primary, secondary, and early latent syphilis the recommended treatment is 2.4 million units of benzathine penicillin, intramuscularly in a single dose. ${ }^{26}$ The local compliance for three doses of penicillin, as is routinely recommended, is less than $50 \%$, and is not free of charge.

Some authors in different settings such as Canada, ${ }^{19}$ France, ${ }^{20}$ the United States, ${ }^{27} \mathrm{Fiji}^{28}$ and South Africa ${ }^{29}$ are questioning the efficacy of penicillin in a single dose and are recommending two to three doses. A recent prospective study performed by Alexander et $a l^{30}$ concluded that the CDC recommended regimens are effective, but the highest risk of fetal treatment failure exists with maternal secondary syphilis.

In conclusion, the proposed approach of on site testing and treatment by the nurse midwives is feasible and it was possible to decrease the perinatal mortality as well as maternal seropositivity at delivery.

Contributors: NBO had the main responsibility for the study design, implementation, data analyses, and writing of the manuscript; KC contributed in study design and data analysis; $\mathrm{EF}$ had the responsibility for all the serological tests done in the laboratory and participated in the data analysis; MC did all the field work, data computerisation, and participated in data analysis; SB had responsibility of supervising all phases of the study.

1 Temmerman M, Mohamedali F, Fransen L. Syphilis prevention in pregnancy: an opportunity to improve repro-
ductive and child health in Kenya. Health Policy and Planning 1993;8:122-7.
2 McDermott J, Steketee R, Larsen S, et al. Syphilis. associated perinatal and infant mortality
Bull World Health Organ 1993;71:773-80.

3 Wilkinson D, Sach M, Cannolly C. Epidemiology of syphilis in pregnancy in rural South Africa: opportunities for control. Trop Med Int Health 1997;2:57-62.

4 Opai-Tetteh ET, Hoosen AA, Moodley J. Re-screening for syphilis at the time of delivery in areas of high prevalence. $S$ Afr Med f 1993;83:725-6.

5 Bam RH, Cronjé HS, Muir A, et al. Syphilis in pregnant patients and their offspring. Int $\mathcal{F}$ Gynecol Obst 1994;44: 113-8.

6 Hira SK, Bhat GJ, Chikamata DM, et al. Syphilis intervention in pregnancy: Zambian demonstration project. Genition in pregnancy: Zambian
tourin Med 1990;66:159-64.

7 Rutgers S. Syphilis in pregnancy: a medical audit in a rural district. Cent Afr f Med 1993;39:248-53.

8 Liljestrand J, Bergström S, Nieuwenhuis F, et al. Syphilis in pregnant women in Mozambique. Genitourin Med 1985;61: 355-8

9 Cossa HA, Gloyd S, Vaz RG, et al. Syphilis and HIV infection among displaced pregnant women in rural Mozambique. Int f STD AIDS 1994;5:117-23.

10 Vuylsteke B, Bastos R, Barreto J, et al. High prevalence of sexually transmitted diseases in a rural area in Mozambique. Genitourin Med 1993;69:427-30.

11 Hederstedt B, Liljestrand J, Bergström S, et al. IgMantibodies against $\mathrm{T}$ pallidum detected in sera from mothers of stillborn babies in Mozambique by the solid-phase haemadsortion assay (SPHA). Int $\mathcal{F}$ STD AIDS 1992;3: 347-9.

12 Bique Osman N, Folgosa E, Gonzalez C, et al. Genital infections in the aetiology of late fetal death: an incident case-referent study. $\mathcal{F}$ Trop Ped 1995;41:258-66.

13 Folgosa E, Osman NB, Gonzalez C, et al. Syphilis seroprevalence among pregnant women and its role as risk factor for stillbirth in Maputo, Mozambique. Genitourin Med 1996;72:339-42.

14 Lindstrand A, Bergström S, Bugalho A, et al. Perinatal transmission of parturient syphilis in Mozambique. Int 7 STD AIDS 1995;6:709.

15 Lindstrand A, Bergström S, Bugalho A, et al. Prevalence of syphilis infection in Mozambican women with second trimester miscarriage and women attending antenatal care in second trimester. Genitourin Med 1993;69:431-3.

16 Jenniskens F, Obwaka E, Kirisuah S, et al. Syphilis control in pregnancy: decentralization of screening facilities to primary care level, a demonstration project in Nairobi, primary care level, a demonstration project in Nairo

17 Delport SD, Berg JHY. On-site screening for maternal syphilis at an antenatal clinic. $S$ Afr Med f 1998; 88:43-4.

18 Wilkinson D, Sach $M$. Improved treatment of syphilis among pregnant women through on-site testing: an intervention study in rural South Africa. Trans $R$ Soc Trop Med Hyg 1998;92:348.

19 Ray JG. Lues-Lues: maternal and fetal considerations of syphilis. Obstet Gynecol survey 1995;50:845-50.

20 Narducci F, Switala I, Rajabally R, et al. Syphilis maternelle et congenitale. 7 Gynecol Obstet Biol Reprod 1998;27:15060.

21 Hurtig AK, Nicoll A, Carne C, et al. Syphilis in pregnant women and their children in the United Kingdom: results from national clinician reporting surveys 1994-7. BMF 1998;317:1617-9.

22 Welch J. Antenatal screening for syphilis. Still important in preventing disease. BM7 1998;317:1605-6.

23 Sheffield JS, Wendel Jr GD. Syphilis in pregnancy. Clin Obstet Gynecol 1999;42:97-106.

24 Mobley JA, McKeon RE, Jackson KL, et al. Risk factors for congenital syphilis in infants of women with syphilis in South Carolina. Am F Publ Health 1998;88:597-602.

25 Kambarami RA, Manyame B, Macq J. Syphilis in Murewa district, Zimbabwe: an old problem that rages on. Cent Afr 7 Med $1998 ; 44: 229-32$.

26 Centers for Disease Control and Prevention. 1998 Guidelines for treatment of sexually transmitted diseases. MMWR 1998;47:8-38.

27 Hollier LM, Cox SM. Syphilis. Sem Perinatol 1998;22:32331.

28 Schramm M. Syphilis resurgent. Aust NZ f Obstet Gynaecol 1997;37:377-82.

29 Donders GG, Desmyter J, Hooft P, et al. Apparent failure of one injection of benzathine penicillin $\mathrm{G}$ for syphilis during pregnancy in human immunodeficiency virus-seronegative African women. Sex Transm Dis 1997;24:94-101.

30 Alexander JM, Sheffield JS, Sanchez PJ, et al. Efficacy of treatment for syphilis in pregnancy. Obstet Gynecol 1999;93: $5-8$. 